\title{
Continuum Scattering and Formation of Emission Lines
}

\author{
Nikoghossian A.* \\ NAS RA V.Ambartsumian Byurakan Astrophysical Observatory, Byurakan 0213, Aragatsotn Province, Armenia
}

\begin{abstract}
The effect of radiation scattering in continuum on the spectral lines formation is treated. Compared to the early works in this direction, the conditions of emission lines appearance in the atmosphere with the temperature gradient with depth is discussed in detail. For simplicity's sake, the coherent scattering in the spectral line frequencies is adopted. By the example of atmospheres of supergiant stars of type A and adjacent classes, it is shown which lines and in what parts of the spectrum can be observed in the emission.
\end{abstract}

\section{Introduction}

This article continues our study (Israelian \& Nikoghossian, 1996) on the effects of continuum scattering on spectral line formation. Besides the purely theoretical meaning, the problem is important in interpreting spectra of non-stationary stars of different types at some stages of their evolution. The concrete implication of this study concerns supergiants of type A and adjacent classes with extended atmospheres. The high degree of ionization of hydrogen, partly helium, due to high temperature and low gas density, provides a sufficient number of free electrons to make the effect of electron scattering quite measurable (Owocki et al., 2004, Wolff, 1983).

In the first part of this study (Nikoghossian, 2020), we reviewed in detail the effect of this scattering on the brightness curve and the hydrogen spectrum jumps of these objects. The main purpose of this work is to determine the conditions under which the emission line formation Shuster's mechanism operates (Schuster, 1905). The problem was considered by various authors under different initial assumptions (see, e.g. Gebbie \& Thomas, 1968, Hummer \& Rybicki, 1967). The LTE nd Non-LTE models of the problem were discussed. Of particular importance in the problem is a more accurate account of the temperature gradient in the atmosphere. This problem was solved by Harington but for the grey atmosphere. In this paper we adopt a simpler approach to the problem, which is based on Ambartsumian's principle of invariance (Ambartsumian, 1943, 1960) and on some other results we obtained in this direction.

\section{The simplest problem of coherent scattering in both the contin- uum and spectral line}

Before addressing the problem under study, let us first consider the auxiliary problem and introduce the values that control the scattering processes in the atmosphere.

Consider the 1D semi-infinite isothermal atmosphere absorbing and scattering in both the continuum and spectral line, the boundary of which is illuminated by the continuum radiation of unite intensity. Photons falling on the medium can be absorbed and scattered both in the continuum and within the spectral line. By denoting the densities of neutral atoms and electrons respectively through $n_{1}$ and $n_{e}$, for the probability of the photon scattering in the continuous spectrum, $p_{1}$, we can write

$$
p_{1}(\nu)=\frac{n_{e} \sigma_{0}}{n_{1}\left(\chi_{\nu}+\kappa_{\nu}\right)+n_{e} \sigma_{0}},
$$

*nikoghoss@yahoo.com 
where the following notations are used: $\chi_{\nu}$ and $\kappa_{\nu}$ are the coefficients of absorption in the continuum and spectral line, respectively, per hydrogen atom. We adopt the two - level model, so that the atoms are either ionized or are in the ground state. The electron scattering coefficient $\sigma_{0}$ is expressed through electron's classical radius to yield

$$
\sigma_{0}=\frac{8 \pi}{3}\left(\frac{e^{2}}{m c^{2}}\right)^{2}
$$

where $m$ and $e$ are the mass and the charge of electron, and $c$ is the light speed. Assuming that the number of free electrons is due solely to the ionization of hydrogen atoms, Eq.(1) can be rewritten in a more compact form

$$
p_{1}(x)=\frac{\bar{\gamma}}{\alpha(x)+\beta+\bar{\gamma}}
$$

where the following notations are introduced $\bar{\gamma}=\left(n^{+} / n_{1}\right)\left(\sigma_{0} / \kappa_{0}\right), n^{+}$is ions density, and $\kappa_{0}$ is the line absorption coefficient at the central frequency, $\alpha(x)$ is the line absorption profile, $x$ is the dimensionless frequency measured by displacement from the center of the line in Doppler widths and $\beta$ is the ratio of the continuum absorption coefficient to that in the center of the line.

By an analogous manner, for the probability of a photon to be scattered in the line denoted by $p_{2}$, we may write

$$
p_{2}(x)=\frac{\lambda \alpha(x)}{\alpha(x)+\beta+\bar{\gamma}}
$$

where $\lambda$ is the coefficient of the quantum re-radiation in the elementary event of scattering in the line. The frequency redistribution function consists of two components, $r_{1}=\alpha(x) \delta\left(x-x^{\prime}\right)$ and $r_{2}=\bar{\gamma} \delta\left(x-x^{\prime}\right)$, correspondingly for the spectral line and continuum (here $\delta$ is Dirac's delta function). It is easy to see that these two scattering processes can be combined and considered as a single diffusion process with a scattering coefficient $\tilde{\lambda}$, where

$$
\tilde{\lambda}(x)=\frac{\lambda \alpha(x)+\bar{\gamma}}{\lambda \alpha(x)+\beta+\bar{\gamma}}
$$

The explicit expression obtained with use of invariance principle for the reflection coefficient for monochromatic scattering (see, e.g., Sobolev 1963), has a form

$$
\rho(x)=\frac{1}{\tilde{\lambda}}(2-\tilde{\lambda}-2 \sqrt{1-\tilde{\lambda}})
$$

which in view of Eq.(5) after some simple algebra yields

$$
\rho(x)=\frac{\sqrt{v(x)}-\sqrt{u(x)}}{\sqrt{v(x)}+\sqrt{u(x)}}
$$

where, for brevity, the following notations are introduced: $v(x)=\alpha(x)+\beta+\bar{\gamma}, u(x)=(1-\lambda) \alpha(x))+\beta$. The function $\rho(x)$ has probability meaning and gives the profile of radiation reflected from semi-infinite isothermal atmosphere denoted hereafter by $R(x)$. We will not discuss this auxiliary result and proceed directly to the problem under study.

\section{The atmosphere with the temperature gradient}

It is well known (see, for instance Mihalas, 1978, Sobolev, 1963) that the problem of the spectral lines formation in the Milna-Eddington model with allowance for the absorption in continuum is reduced to the solution of the transfer equation with a source term, describing the distribution of primary energy sources, of the following form

$$
\varepsilon(\tau, x)=u(x) B_{\nu}[T(\tau)]
$$



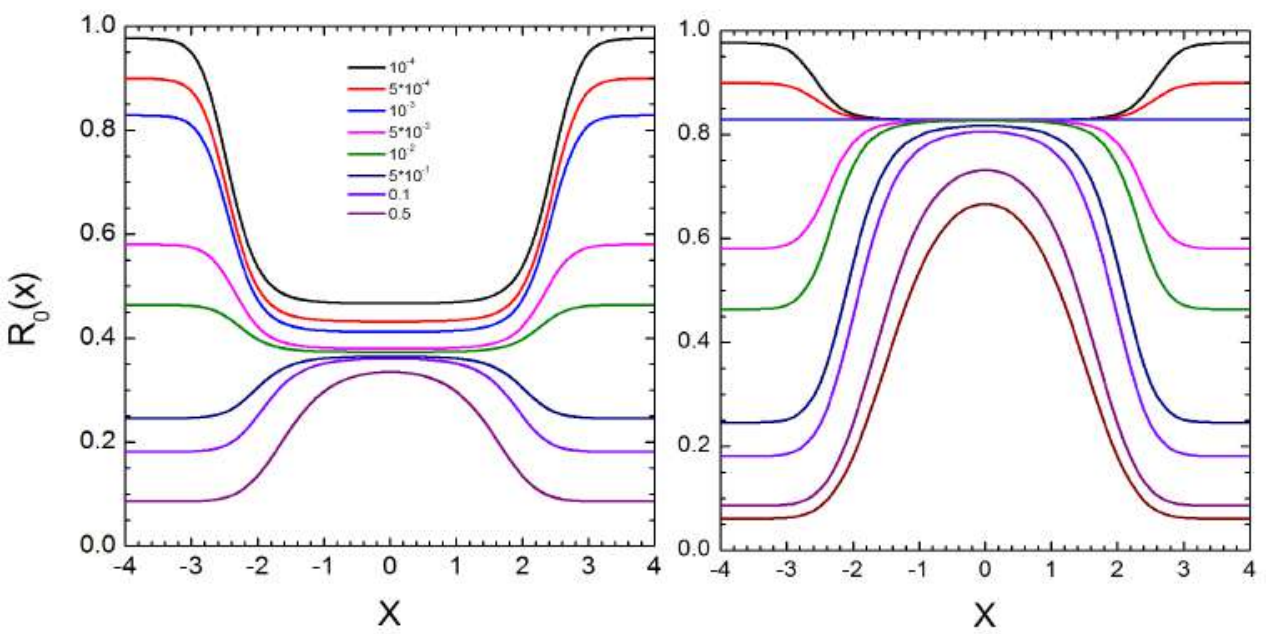

Figure 1. The line profiles formed in an isothermal atmosphere for $\lambda=0,9$ (left) and $\lambda=0.5$ (right) for indicated values of $\bar{\gamma}$. In both cases $\beta$ is $10^{-} 3$

where $B_{\nu}(T)$ is the Planck function depending through temperature on optical depth in the center of the line. In solving the considered problem it is usual to make use of an expansion of the Planck function in the form of a series in terms of $\tau$, as given by

$$
B_{\nu}[T(\tau)]=\sum_{n=0}^{\infty} \frac{B_{\nu n}}{n !}(\beta \tau)^{n} .
$$

In our papers (Haruthyunian \& Nikoghossian 1978, Nikoghossian \& Haruthynian 1979) we suggested an easy way of determining spectral line profiles formed in atmospheres with distributed in it energy sources of the form Eq.(9). It has been shown that line profiles for arbitrary internal sources of the form Eq.(8) are expressed in a recursive way in terms of the profile formed in an isothermal atmosphere with $\varepsilon=u(x)$. Note that $u(x)$ also has a probabilistic meaning by giving the probability that the line photon will undergo true absorption and thermalized either in the line frequencies or in the continuum. In the first of the above works we have shown that there is a simple relationship between the line profile formed in the isothermal atmosphere, $R_{0}$, and the reflected line profile $R_{*}$.

$$
R_{*}(x)+R_{0}(x)=1 .
$$

This relationship has a plain probabilistic interpretation and follows from the fact that the radiation incident on the medium is either reflected from it or is thermalized in the course of multiple scattering in it. Now with use of Eq.(7), we find

$$
R_{0}(x)=2 /\left(1+\frac{1}{\sqrt{u(x) / v(x)}}\right) .
$$

Consider the question under what conditions in this simple case of isothermal atmosphere the spectral line can appear in the emission. Obviously, it requires that $R_{0}(x)>R_{0}(\infty)$. Eq.(11) yields

$$
(1-\lambda) \bar{\gamma}>\lambda \beta \text {. }
$$

In the simplest case of isothermal atmosphere, Eq. (12) is a necessary and sufficient condition for the spectral line to appear in the emission. At least two important conclusions can already be drawn on the base of Fig. 1 We see that this condition will be satisfied above all by weak lines, which, on an average, are formed in deeper layers of the atmosphere. This emphasizes the importance of taking into account the temperature gradient in it. In addition, as might be expected, the degree of ionization, on which the density of free electrons depends, plays an important role in the appearance of emission lines. 

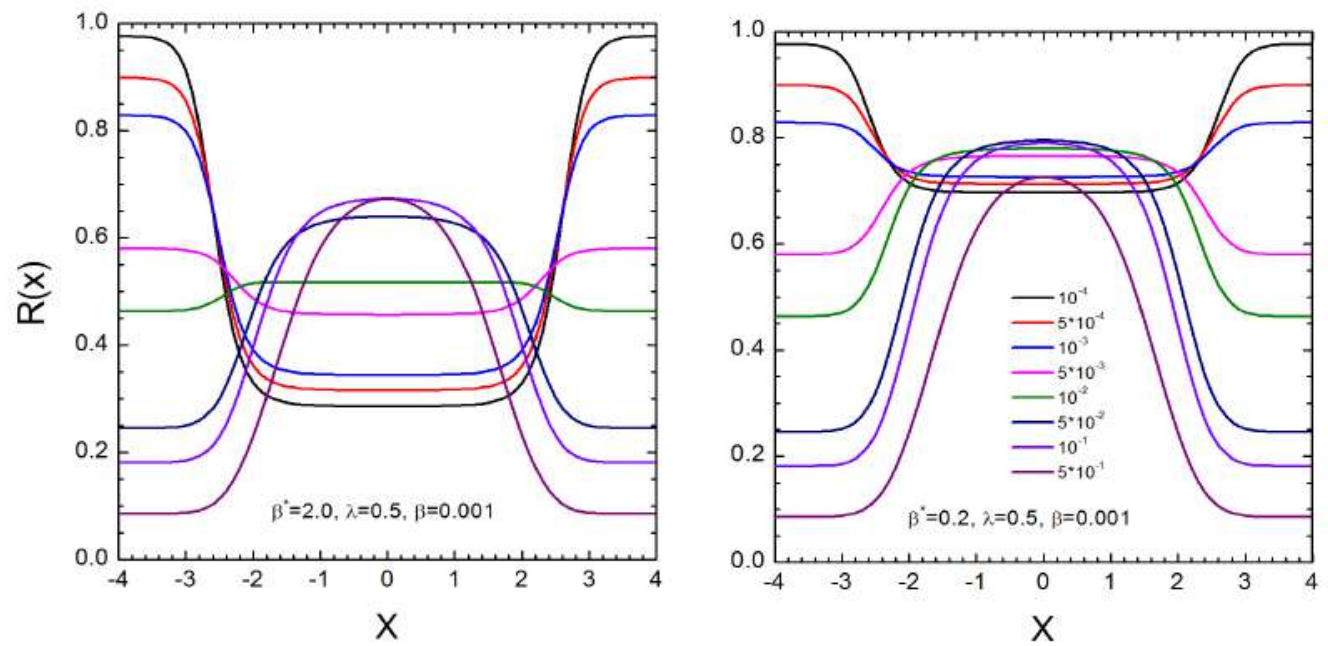

Figure 2. The profiles of spectral lines with $\lambda=0,5$ formed in the atmosphere with temperature gradient in the presence of electron scattering. The lines of two extreme domains of continuum spectrum are depicted

We give some estimates for the degree of ionization of hydrogen for temperatures about $10^{4}-10^{5} \mathrm{~K}$ and spectral lines with moderate values of $\lambda \leq 0,8 \div 0$, 9in a wide range of wavelengths 300-900nm. We have $\gamma=\sigma_{0} / \kappa_{0}$, where $\sigma_{0}=6.65 \cdot 10^{-} 25 \mathrm{~cm}^{2}$ and $\kappa_{0}$ for the pure Doppler broadening of the line is given by (see,e.g., Mihalas, 1978, Sobolev, 1963)

$$
\kappa_{0}=\frac{\lambda_{0}^{3}}{8 \pi^{3 / 2} v} \frac{g_{k}}{g_{i}} A_{k i}
$$

where $\lambda_{0}$ is the wavelength of the line center, $A_{k i}$ are Einstein's coefficients of atomic spontaneous transitions and $v=\sqrt{2 k T / M}$ is the mean thermal velocity (for other quantities the usual designations are adopted). To estimate the order of magnitude for the degree of ionization, note that the right side of the inequality (12) at the chosen numerical values of parameters is the value less than $10^{-4}$, while the ratio $\sigma_{0} / \kappa_{0}$ for the lines with $A_{k i}$ of about $10^{5} \div 10^{6} \mathrm{sec}^{-1}$ lays in the interval $10^{-9} \div 10^{-10} \mathrm{sec}^{-1}$. This means that the degree of ionization of the order of $10^{5}$ and above is sufficient for the appearance of emission lines resulting from transitions between the upper energy levels. Such a high degree of ionization may well occur in the high-temperature and rarefied shells of the stars under consideration.

Let us turn now to the effect of the temperature gradient. In a linear approximation, we have

$$
B_{\nu}(T)=B_{\nu}\left(T_{0}\right)\left(1+\beta_{\nu}^{*} \bar{\tau}\right),
$$

where $\bar{\tau}$ is the optical depth in continuum in terms of Planck's mean coefficient of absorption, and

$$
\beta_{\nu}^{*}=\frac{3}{8} \frac{h \nu}{k T_{0}}\left(1-\mathrm{e}^{-\frac{h \nu}{k T_{0}}}\right)^{-1} .
$$

In accordance with Haruthyunian \& Nikoghossian (1978), for the normalized profile of the line, we have

$$
R(x)=[P(x) / P(0)] R_{0}(x),
$$

where

$$
P(x)=1+\frac{\beta_{\nu}^{*} \beta}{v(x) \sqrt{1-\tilde{\lambda}(x)}}=1+\frac{\beta_{\nu}^{*} \beta}{\sqrt{v(x) u(x)}} .
$$

The transition from the center of the line to its wings in functions $R_{0}(x)$ and $P(x)$ occurs in mutually opposite directions, and there is not any closed-form formula for conditions of the line appearance in emission. 

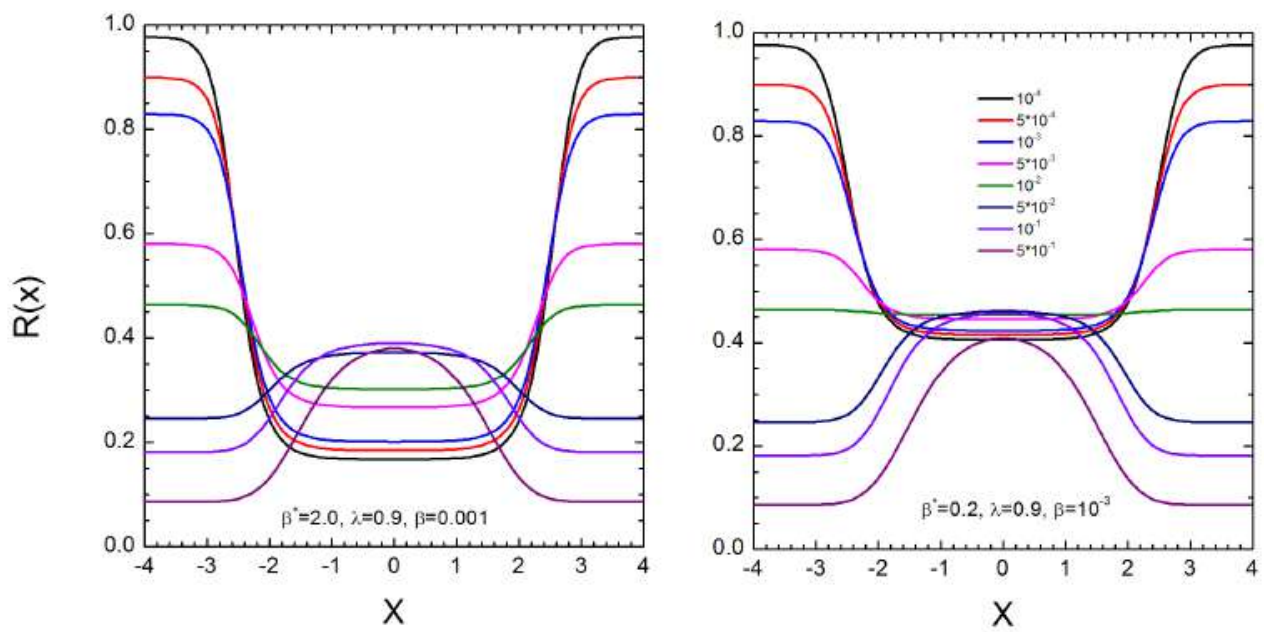

Figure 3. The same as in Fig. 2 for $\lambda=0,9$

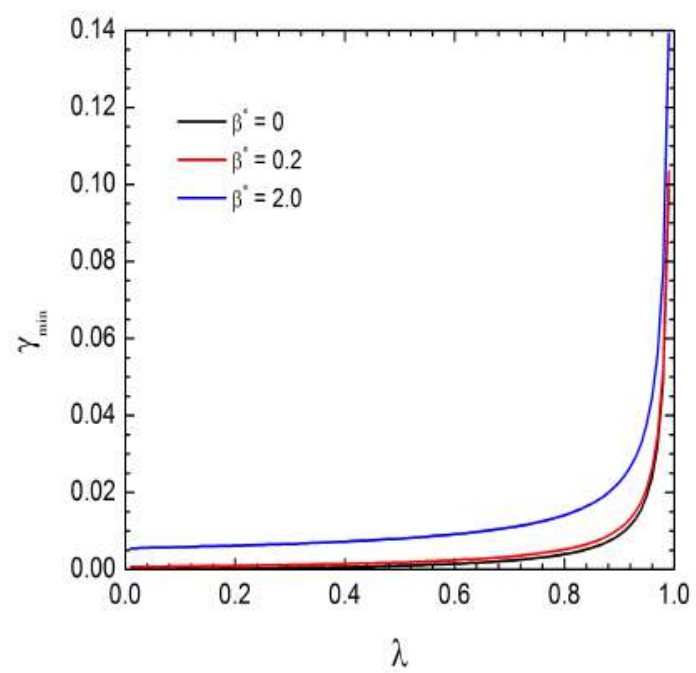

Figure 4. Dependence of threshold values of $\bar{\gamma}$ on the coefficient of scattering within a line. 
Figs.2,3 show the line profiles formed in non-isothermal atmospheres with a linearly growing with depth temperature. Two cases corresponding to two values of the coefficient $\beta_{\nu}^{*}$ which refer to two different (shortwave and long-wave) spectrum domains are considered. We see that, as in the isothermal atmosphere, the threshold for the influence of electron scattering on the spectral lines here also depends to a large extent on the $\lambda$ value. This is particularly well illustrated in Fig.4, which shows the dependence of the lower threshold of the scattering action in continuum resulting appearance of the lines observed in emission.

The specified threshold also essentially depends on value $\beta_{\nu}^{*}$, that is, on what area of a continuous spectrum the line is formed. Taken together, these facts allow us to conclude that the most probable is the appearance of emission in relatively weak lines in the long-wave regions of the spectrum. In all, the differences between the line profiles formed in an isothermal atmosphere and in an atmosphere with a temperature gradient are quantitative and mainly repeat each other. Profiles of both absorption and emission lines basically are broadened and bell-shaped, which become narrower with the increasing role of electron scattering. The latter is due to the fact that these lines, on an average, are formed in more superficial and relatively low-temperature layers of their formation.

The results presented in the paper show that despite the comparatively small coefficient of electron scattering, under certain physical conditions spectral lines can be observed in the emission. At the same time, the line profiles have a form characteristic of electron scattering. However, in some cases it may be necessary to address the problem in a more general formulation, taking into account the frequency redistribution within the line. At the same time, it is important to take into account the dynamics of the medium in the line formation, so when interpreting it, problems similar to that we treated should be combined with one or another dynamic models.

\section{References}

Ambartsumian V. A., 1943, Dokl. Acad. Nauk SSSR, 38, 257

Ambartsumian V. A., 1960, Scientigic Papers, 1, Izd. AN ArmSSR, Yerevan

Gebbie K. B., Thomas R. N., 1968, ApJ, 154, 285

Harrington J. P., 1970, ApJ, 162, 913

Haruthyunian H. A., Nikoghossian A. G., 1978, Dokl. Acad. Nauk SSSR, 242, 66

Hummer D. G., Rybicki G., 1967, Methods in Computational Physics, 7, 53

Israelian G., Nikoghossian A., 1996, JQSRT, 56, 509

Mihalas D., 1978, Stellar atmospheres

Nikoghossian A., 2020, ComBAO, 67, 1

Owocki S. P., Gayley K. G., Shaviv N. J., 2004, ApJ, 616, 525

Schuster A., 1905, ApJ, 21, 1

Sobolev V. V., 1963, A treatise on radiative transfer.

Wolff S. C., 1983, The A-Stars: Problems and Perspectives. NASA SP-463. Vol. 463 